\section{Neuzeitliches aus der deutschen Feinkeramik, unter besonderer Berücksichtigung der Herstellung von Porzellan- und Steinzeugnotgeld.}

Von W. Funk, Meißen.

(Vorgetragen in der Hauptversammlung des Bezirksvereins Sachsen-Thuringen am (Eingeg. 15.11. 1922.)

Als Zweiggebiet der angewandten Silicatchemie birgt die Keramik, die Tonwarenindustrie, für den Chemiker eine Fülle von Arbeitsmaterial. Die keramische Industrie, die in der ersten Hälfte des 19. J.hrhunderts in überwiegender Weise noch mit empirischen Mitteln arbeitete, ist in den letztvergangenen fünfrig Jahren nach und nach immer tiefer vom Geiste der chemischen Wissenschaft durchdrungen worden. Eine stetig wachsende Zahl von Chemikern hat sich während dieser Zeit in den Dienst der Tonwarenindustrie gestellt, und dies hat - wir können das ruhig sagen, ohne ruhmredig zu erscheinen diecer Indu-trie einen gewultigen Nutzen gebracht, sowohl in technischer Hinsicht, d. h. in bezug auf die Schaffung neuer keramischer Massen, neuer Dekorationsverfahren und andere Forts'hritte, als auch - oder richtiger: und infolge hiervon - in wirtschaftlicher Beziehung. durch Erschließung neuer Anwendungsgebiete und Erhőhung des Warenabsatzes. Selbstverständlich gebührt auch dem Maschineningenieur an diesem Aufblühen der keramischen Industrie ein unb* streitbares Verdienst, das ihm keineswegs vorenthalten werden soll. Denn erst dadurch, daß er die erforderichen maschinellen Hilfsmittel schuf, war es möglirh, alle diejenigen Fabrikationsverbesserungen einzufïhren, welche lür eine großzingige Herstellung zweckentsprechender erstklassiger Waren unbedingt notwendig sind.

Wer von Ihnen eine grolere Reihe keramischer Fahriken, von der Ziegelei bis zur Porzellanfabrik, in den verschiedenen Teilen Deutschlands besur'ht hat, der wird vielleicht - nicht ohne jede Berechtigung - darauf hinweisen wollen, dati trotz der von mir soeben angedeuteten fortgeschrittenen Entwicklung in dieser Industrie norh manches verbesserungsbedurftig ist Aber es handelt sich in solchın Fällen meist um kleinere Werke älteren Ursprungs oder in abgelegenen Gegenden, denen eine ungleich größere Zahl moderner oder ge-chickt modernisierter Fabriken gegenübersteht, die sich unter der sachverständigen und durchaus neuzeitlichen Leitung einzelner Besitzer oder von Aktienunternehmungen befinden, und bei denen das vorhin von mir Gesagte zweifellos zutrifft.

Diese mit der Zeit fortgeschrittenen Fabriken, die heute bei weitem die überwiegende Mehrzahl bilden, haben ihren keramischtechnischen Hor-hstand vor allem einer längeren und oft mühsamen, zielbewuBten Organisationsarbeit zu verdanken. Es ist überhaupt das eigentlimliche der keramisehen Industrie, daß3 man bei ihr im praktischen Betriebe Neuerungen nur allmählich durchführen kann, wenn nicht Störungen entstehen sollen. Denn in kaum einer anderen Industrie erfordert der Fabrikationsgang so viel räumlich und zeitlich völlig voneinander getrennt verlaufende Einzelprozesse wie in der Keramik, besonders in der Feinkeramik. Will man sich nicht mit einem bloßen Laboratoriumsversuche in kleinem $M_{a}$ B.tabe begntigen, so dauert es unter ('mständen wochen- oder monatelang, bis man das Ergebnis einer Fatırikationsänderung in der Hand hält, um diese Änderung auf ihre $Z$ weckmäßigkeit prufen zu können. Das liegt in der Eigenart des keramischen Fabrikationsganges begrindet, der infolge der verschiedenen den ein zelnen Werkstoffen und $Z$ wischenprodukten aufzuerlegenden Bearbeitungsphasen vielfach so langsam und umständlich sein muß.

War es . nun früher ein bloßes "Probieren", das man den notwendig werdenden Fabrikationsverbesserungen' zugrunde legte, so ist man mit dem Aushau der analytischen Chemie und der physikalisch-chemischen Materialprufungsmethoden immer mebr zum ${ }_{n}$ Studieren ${ }^{*}$, d. h. zum wissenschaftlichen Erforschen und Kontrollieren der einzelnen Fabrikationsprozesse, übergegangen, und zwar mit ausgezeichnetem Erfolge. Dahei ist es gleichgült $g$, ob es sich um grobkeramische oder feinkeramische Fabrikation handelt. Zur Grobkeramik rechnet man, wie ich zur Erläuterung einflechten möchte, alle keramischen Erzeugnisse von grobkörnigem Gefüge und unreiner, nicht einhritlicher Farbe, ganz gleich, ob die Masse na. $h$ dem Brennen noch poros oder schon dichtgebrannt ist, also vor allem die Mauer- und Dachziegel, die feuerfesten Steine und andere Sthamottewaren, wie Gasretorten, Glashäfen usw., dınn das g wơhnlirhe Tơpfergeschirr, das technische SLeinzeug, z. B. die Kanalisationsrohre, Fußboden- und andere. Klinkerplatten und manches andere mehr. Zur Feinkeramik hingegen grborien ille Tonwaren, die einheitliche, reine Farbe und frinkörnig poröse bis gla ig dichte Besrhaffenheit zeigen, also das Steingat in seinen verschiedenen Abarten, das feine Steinzeug und das Forcellan.

Angew. Cbemie 1922. Nr. 14.
Wir haben uns hier fast ausschließlich mit der Feinkeramik zu beschäftigen, die bei uns in Deuts'bland auf boch entwickelter Stufe steht, aber aus sich heraus einer noch böheren Entwicklung zustrebt, um qualitativ immer besser und dabei doch billiger fabricieren zu können.

Die Nơte der Zeit haben in den verscbiedenen Zweigen der Industrie allgemein einen weit engeren Zusammenscblul' der einzelnen Fabriken gleicher Art herbeigeführt als er vor dem Kriege bestand. Kleine Gegensätze haben länłst zurücktrelen müssen vor den großen wirtschaftlicben Fragen, die alle in gleichem Maße berübren und bis ins Innerste bewegen. Als solche sind vor allem zu nennen die Arbeiterirage, die Geldentwertung, die Regelung der Verkaufspreise im In- und Ausland, die Kohlenfrage, die Rohstofffrage und manche andere.

In der Erörterung und Lösung solcher wichtiger Fragen geht nun auch die keramische Industrie geschlossen vor, und zwar nicht nur zur unmiltelbaren Verfolgung gemeinsamer rein wirtschaftlicher Interessen, sondern auch zur Förderung der Keramik in technischer, wissenschaftlicher und künstlerisrber Beziehung. Zu diesem Zwecke war vom Verband Keramisı her Gewerke in Deutscblind, das ist die grolse wirtschaftliche Vereinigung der verschiedenen Zweige der feinkeramischen Industrie, im Jahre 1913 eine besondere techni ichwissenschaftliche Abteilung begründet worden, die es sich zur Aufgabe machte, "Theorie und Praxis in keramischen Fragen zusammenzuftuhren und die Forschungen der ersteren der letzleren zunutze zu machen“. Dazu war es nötig, daß alle Kräfte sich zu gemeinsamer Arbeit die Hand reichten, jhre Kenntnisse und Erfahrungen austauschten und sich in den Dienst des allgemeinen Interesses stellten.

Diese technisch-wissenschaftliche Abteilung hat auch während des Krieges weiter gearbeitet, wenn auch natürlich unter erschwerten Verhältnissen. Sie ist dann nach dem Kriege im Jahre 1920 in eine selbständige Vereinigung, die „Deutsche Keramische Gesellschaft", umgewandelt worden, der zurzeit über 250 Mitglieder, darunter zehn grotie Fachveıbände und mehr als 85 Firmen, angehören. Die Orgunisation dieser neuen D.K.G., die übrigens im vorigen Jahre in Dresden ihre erste Hauptversammlung abgehalten hat und Ende Juni 1922 wiederum hier tagen will, sieht außer dem Vorstande einen besonderen Leiter der wissenschaftlichen und literarischen Arbeiten und einen mehrkőpfigen Arbeitsausschuß vor. Diese Organe legen die Richtlinien fest, nach denen gearbeitet werden soll, und sorgen dafür, daß die Bearbeitung von Aufgaben, die ein allgemeines Interesse haben, auf möglichst breiter Grundlage vorgenommen wird. Die D.K.G. gibt eigene Tätigkeitsberichte beraus, die auch sonst vieles Neue aus den verschiedensten Zweigen der Keramik bringen, teils auf praktischem, teils auf wissenschaftlichem Gebiete.

Die Bestrebungen der deutschen Keramiker, ibrer Industrie in gemeinsamer Forschungsarbeit zu nützen, sind also recht ernsthafte. Ich will versuchen, in folgendem kurz zu zeigen, welche feinkeramischen Probleme der Bearbeitung zunächst vor allem noch harren.

An erster Stelle steht hier die Frage der besseren Brennstoffausnutzung. Sie ist, wie alle mit dem Brennstoffvelbrauch zusammenhängenden Fragen, für die deutsche keramische Industrie unter den heutigen Veihältnissen von der allergrößten Medeutung. Es ist für diese Industrie geradezu eine Lebensfrage, die voraussichtlich niernals mehr oder doch auf unbestimmt lange Zeit nicht in ausreichender Menge zur Verfägung stehenden hochwertiken Brennstoffe durch andere zu ersetzen. Die Gesicht: punkte ${ }^{1}$ ), unter denen die keramischen Brennverfabren neuerdings kritisch geprüft werden müssen, sind also erstens Umstellung auf einen anderen Brennstoff und zweitens Sparsamkeit.

Man hat zunächst mit der Untersuchung der zurzeit in den einzelnen Fabriken üblichen Brennmetboden begonnen und prüft diese darauf hin, ob bei ihnen rasch durchzuführende Verbesserungen möglich sind und unter Veringerung des Brennstof faufwandes ebenso gute Ware wie bisber erzielt werden kann, oder ob bei besserer Ausbildung der Brennkontrolle und vor allem allgemeinerer Einführung selbsttätiger Kontrollapparate - Pyrometer und Ruubgasprtifer viellicht gar ein noch gleichmäßigeres gutes Brennergebuis möglich ist als früher.

Zur Untersuchung der Wirtschaftlichkeit der keramischen Industrieofen nimmt man gle chzeitig genau koutrollierte Versuchsbrände vor, um mit Hilfe der gelundenen Ér gebnisse eine genaue Wärmeautrechnung - Wärmebilanz - aufstellen zu kơnnen. Man ermittelt also, wieviel der erzeugten Wärme den zu brennenden Waren zugute kommt, wieviel durch den Schornstein, wieviel durch Strahlung verloren geht usw., und erfährt so, wie jeder einzelne Industrieofen arbeitet. Sulche Bilanzrechnungen führt man bekanntlich im Hättenwesen, besonders in der Eisenhültenindustrie, schon seit einer Reihe von Jabren aus.

1) Vgl. hierzu die Ausführungen von E. Reutlinger. Berichte d. D.K.G. 1920 , Bd. 1, Heft 3, S. 20, und ebeuda 1921, S. 33. 
Man erhofft sich von allen diesen in neuester Zeit in die Wege geleiteten Bestrebungen einen großen wirtschaftlichen Nutzen für die kerimische, insbesondere die feirikeramische Industrie.

Der andere Gesicht punkt, den ich vorhin andeutete, nümlich Umstellung auf einen anderen, weniger hochwertigen Brennstoff st: ht in engem Zusammenhange nit der Einführung brauchbarer und mit minderwertigen Brennstoffen arbeitender Gaser feinkeramischer Öfen. Dies ist ein Problem, das sich nicht so rasch lösen läßt, wenn es überhaupt für alle praktischen Fälle jemals zufriedenstellend gelöst werden kann. Diejenigen von Ibnen, welche gehört haben, was ich vor etwa zwei Jahren hier im Verein über die Geschichte und Aussichten der Beheizung feinkeramischer Öfen mit Generatorgas ausgeführt habe, werden sich vielleicht noch erinnern, daß jeh schon damals betonte, wie schwierig und kostspielig die erforderlichen Vorversuche hierzu seien, und wie sehr im einzelnen Falle das Gelingen von den örtlichen Verhältnissen abhängt. Es müssen in der Tat noch viele Versuche gemacht werden, ehe man an eine allgemeine Einführung der Generalorgasfeuerung für das Bremnen aller Arten von Porzellanwaren denken kann. Solche Versuche kosten aber, grnz besonde's heutzulage, sehr viel Geld, und durch einen einzigen mißlungenen Gasbrand - das ist ebenfalls wohl zu bedenken - können bei vollgesetztem Ofen dur'h den entstehenden Warenausfall unter Umständen weit größere Werte verloren gehen als bei einer Gasbrbeizung der Porzellan- und Steingutbrennofen durch Kohlenersparnis gewonnen werden könnten. Auch der Reichskohlenkommissar vertrat auf der vorjährigen Hauptversammlung der D.K.G. den Standpunkt, daß man in der Brennstofffrage der Eigenart jeder einzelnen Industrie Rechnung tragen müsse, und daß in der keramischen Industrie die Lö-ung des Ga-feuerungsproblems wesentlich schwerer sei als beispielsweise in der Glasındustrie, da es sich in ersterer meist um nichtkontinuierliche Öfen handle.

Trotz dieser Schwierigkeiten ist die deutsche Feinkeramik aber mit aller möglichen Energie auch an die Lösung dieser Frage herangegangen. Verschiedene Porzellanfabriken oder Interessengemeinschaften solcher hatien den Bau von Versuchsofenanlagen begonnen, und es steht zu erwarten, daß sie an den Ergebnissen ihrer Versuche aucb die Allgemeinheit teilnehmen lassen.

Einen anderen Teil der keramischen wärmewirtschaftlichen Bestrebungen bildet die Frage der zweckmäßigen Ausnutzung der Abbitze aus den abgebrannten Brennöfen. Um Brennstoff $>$ u sparen, ist man mehr als früher bemüht, aus den ahkühlenden öfen die in ihnen aufgestapelte Wïrme abzusaugen und entweder zur Beheizung der Arbeitsräume oder zum Trocknen von Ton und Kaolin, von frisch geformten Schamottekapseln u. a. zu benutzen. Sie seben also auch hier: rationelle Wärmewirtschaft ist das ernste Gebot der Stunde!

Aber sie ist nicht das einzige Problem, dessen Bearbeitung die deutsche keramische Industrie sich zurzeit angelegen sein läßt. In dem Bestreben, unsere einbeimischen mineralischen Rohst offe möglichst weitgehend und mehr als bisher auszunutzen und sie, wenn möglich, an Stelle der zum Teil noch verwendeten ausländischen Materialien zu setzen, werden zurzeit von den Chemikern der (hemisch-technischen Versuchsanstalt bei der Staallichen Porzellanmanufaktur in Charlottenburg die deutschen feldspathaltigen Gesteine, vor allem die Pegmatite, auf ihre Verwendbarkeit geprüft, um ihnen noch weiteren Eingang in die keramische Industrie zu verschaffen und so die Fahrikation zu verbilligen $\left.{ }^{2}\right)$.

"Scbwieriger ist die Frage der Verwertung jener deutschen Kaoline, die entweder zu mager oder wegen ibres zu hohen Eisengehaltes für feinkeramische $Z$ wecke nicht brauchbar sind ${ }^{2}$ ). Es bandelt sich also darum, die Plastizität dieser Kaoline zu erhőhen und ihren Eisengehalt zu verringern. Für beides sind bis jetzt geeignete, im groben brauchbare Verfahren noch nicht gefunden worden. Versuchen, solche auszuarbeiten, werden Untersuchungen vorausgehen müssen, durch die einerseits eine praktisch verwendbare Methode der Plastizitätsmessung geschaffen und andererseits festgestellt wird, in welcher chemischen, mineralischen und physikalischen Form die färbenden Eisenverbindungen in den Kaolinen und Tonen vorhanden sind.

Inwieweit es möglich ist, durch das kurz vor dem Kriege vom Grafen Schwerin elfundene Verfahren der Elektroosmose Tone und Kaoline in solche von erstklassigen Eigenschaften umzuwandeln, wird zurzeit ebenfalls an verschiedenen Stellen praktisch untersucht, wobei mehrfach recht gute Erfolge zu verzeichnen waren, so daß schon verschiedene Werke das Schlämmverfahren durch Elektroosmose eingeführt haben.

Eine weitere Frage $^{3}$ ) ist die Erforschung der Abhängigkeit der Eigenschaften des Porzellans von seiner Zusammensetzung und Herstellungsweise, d.h. von der Art der Verarbeitung und dem Brennverfahren. Es handelt sich vor allem um eine genaue Materialprüfung, die im staatlichen Materialprüfungsamt in Berlin-Dahlı $m$ ausgeführt wird, und die vor allem für die Herstellung der elektrischen Hochspannungsisolatoren wichtige Ergebnisse zeitigen soll.

Um nur noch ein Problem ${ }^{3}$ ) anzufïhren, das ebenfalls von technisch-wirtschaftlicher Bedeutung ist, sei die Herstellung brauchbarer Porzellane mit niedriger Garbrandtemperatur erwähnt,

2) Vgl. hierzu B. Rieke, Ber. d. D.K.G. 1920, Bd. 1, Heft 2, S. 11 u. 12.

3) Ich folge auch hier den Ausführungen Riekes, Ber. d. D. K. G. 1920, Bd. 1, Heft 2, S. 12 u. 13. die also schon etwa bei Segerkegel 7 oder 8 glasig dicht brennen, d. h. bei etwa $1230-1250^{\circ} \mathrm{C}$. Die Garbrandtemperatur des sonst ub. lichen Hartporzellans liegt dagegen bei Kegel 12-15, das sind $1350-1450^{\circ} \mathrm{C}$. Solche niedriggebrannte Massen sind natürlich nicht für chemisch-technische Porzellane geeignet, wohl aber für Gebrauchsgeschirre gewöhnlicher Art, also für Massenfabrikation. Wenn es gelänge, solche Porzellane herzustellen, so wäre das in wirtschaftlicher hinsicht unbedingt als wertvolle Neuerung zu begrüßen, da man dann viel Brennstoff sparen kann.

Auf andere Probleme, wie die Normalisierung der Laboratoriumsporzellangefaße oder die Vervollkommnung der Methoden der keramischen chemisch-technischen Analyse, kann ich hier aus Zeitmangel nicht eingehen, sondern ich wende mich nunmehr dem zweiten Teile meiner Ausführungen zu.

Wollte ich im ersten Teile auf verschiedene Fragen hinweisen, an deren Lösung sich gewissermaßen Vertreter der gesamten deutschen keramischen Industrie in Form einer Arbeitsgemeinschalt beteiligen, so mochte ich im zweiten Teile einiges Nahere über einen Fabilkationszweig mitteilen, durch den im besonderen die Staatl. Porzellan-Manufaktur in Meißen in letzter Zeit viel von sich reden gemacht hat, nämlich die Herstellung von Böttgersteinzeug und vor allem von keramischem Notgeld. Die Staatl. Porzellan-Manufaktur will nicht, wie ich von vornherein betonen möchte, als Erfinderin der Münzen aus keramischem Material gelten, sondern sie nimmt lediglich das Verdienst in Anspruch, durch Schaffung besonderer, den Eigenschalten des keramischen Werkstoffs angepaßter Entwürfe für die neuen Münzen auch auf diesem Gebiete „die Möglichkeit künstlerischkulturellen Schaffens nachgewiesen zu haben “.

In der Tat ist der Gedanke, keramische Münzen herzustellen, keineswegs neu. Das erste Porzellangeld ist in China, dem eigentlirhen Urisprungslande des Porzellans, hergestellt und in Umlauf gebracht woiden. Dieses ostasiatische Porzellangeld war jahrbundertelang im Verkehr und soll in Siam erst vor etwa 30 Jahren außer Kurs geset,t worden sein

Die erste Mitte lung über deutsche Porzellanmünzen ${ }^{4}$ ) stammt aus dem Jahre 1793 und wurde im ,Journal des Luxus und der Moden" veröffentlicht. Sie lautet folgendermaßen:

\section{„Herrn Medailleurs Franz Joseph Eß zu München,}

Medaillen- und Münz-Abdrücke en Biscuit.

Die Erfindung des geschickten Medailleurs EB in München, Münzen und Medaillen mit ihrer Vor- und Kehrseite, also die ganze Münze in ihrer wahren Größe und Form in einer schönen weißen und harten Biscuit-Masse zu liefern, ist für die Kunst sowohl als für das Studium der Geschichte zu interessant, und in Deutschland noch zu wenig bekannt, als daß wir nicht etwas davon sagen, und sie nach Verdienste loben und empfehlen sollten. Diese Mür zabdrücke sind so sauber und scharf, schön weiß, und so hart, wie jedes gute Porzellan en Biscuit; ja man kann sie, wenn sie unrein und schmutzig geworden sind, am besten wieder durch Ausbrennen in einem Töpferofen reinigen. Herr M. E $B$ gibt in dem 2 Bogen starken Verzeichnisse und Preisliste seines Vorrats folgende ausführliche Nachricht davon, die wir für Liebhaber hier einrïcken.

Der Preiß dieser schönen Abdrücke ist dabey so äußerst billig und wohlfeil, daß man sonst gewơhnlich kaum schlechte Gipsabdrücke dafür haben kann."

An diese Mitteilung schliept sich ein von dem Erfinder mit erklärenden Bemerkungen versehenes "Neu vermehrtes Verzeichnis verschiedener zur Kirchen-, Reichs- und Völkergeschichts-Erläuterung dienende Medaillon- und Münz-Abdrúcke, welche bey mir Endesgesetzten gegen die beygesetzten geringen Preiße, jedoch nicht einzeln, $\mathrm{zu}$ baben sind".

Kurz nach Beginn des 19. Jahrhunderts tauchen auch in der Meißner Porzellanmanufaktur die ersten Porzellanmünzen auf, von denen noch Stücke in der der Manufaktur seit dem Jahre 1916 angegliederten Schauhalle aufbewahrt werden

Bei beiden genannten, um das Jahr 1800 unternommenen Versuchen hat es sich nicht um kursfähiges Geld, sondern um die getreue Wiedergabe seltener Münzen für Sammler und Museen gehandelt. Wie "The British Clayworker" vom 15 . September 1920 mitteilt ${ }^{4}$ ), sind in England schon seit dem Jahre 1801 keramische Münzen als Kleingeldersatz für den örtlichen Gebrauch mehrfach verwendet worden.

Die im Verlauf des Weltkrieges von verschiedenen deutschen keramischen Fachmännern unternommenen Versuche, Geldmünzen aus keramischem Werkstoff herzustellen, führten zu keinem oder nur za einem 8rtlich sehr eng begrenzten Erfolge, d. h. die Geldmünzen fanden meistens nur Verwendung als Fabrikkantinengeld o. dgl.

Der Plan, solche Geldstürke auch in den offentlichen Veıkehr einzuführen, ist kurz nach dem Kriege erst der sächsischen Staatsmanufaktur geglückt. Man spricht in der Öffentlichkeit meist vom Meißner Por zellangeld. In Wirktichkeit fahrizieren wir in Meißen weniger Geld aus PorzelJan, sondern meistenks solches aus Steinzeug, das bekanntlich ebenfalls eine völlig dichtgebrannte Masse darstellt wie das Porzellan, aber, gegen das Licht gehalten, nicht durchscheinend, also nicht so glasig ist wie Porzellan.

Die rotbraune, unglasierte Steinzeugmasse, die wir in Meißen verwenden, ist dem alten Böttgersteinzeug möglichst genau nach-

\footnotetext{
4) Vgl. Keramische Rundschau 1920, Nr. 42, S. 435 .
} 
gebildet, d. h. dem Steinzeug, das der europäische Erfinder des Porzellans, Joh. Friedr. Böttger, vor dem Porzellan hergestellt hatte Besondere interessante Einzelbeiten wüßte ich Ibnen über diese Steinzeugmasse nicht mitzuteilen. Wer etwa von thnen solehes Steinzeug herstellen will, der braucht es nur wie wir zu machen, nämlich ein echtes Stück altes braunes Bottgersteinzeug herzunehmen, die Masse zu analysieren, sich die geeigneten Rohstoffe, also roten Ton usw., zu verschaffen und auf Grund des Analysenergebnisses und der $\mathrm{Zu}$ sammensetzung der vertügbaren Rohstoffe eine neue Masse auszuarbeiten. Sie werden dann nach einigem Probieren zu einer Steinzeugmasse gelangen, die denselben matten Glanz und den gleichen angenehm rotbraunen Farbton zeigt wie die gut gelungenen echten alten von Böttger herrührenden Stücke.

Nach den orientierenden Vorbereitungcarbeiten galt es, die neuzeitliche Böttgersteinzeugbereitung auf eine Grundlage zu stellen, auf der eine sichere Massenfabrikation möglich war, wie sie für die Gelderzeugung allein in Frage kam. Besonderes Gewicht mußte auf den Brennprozeß gelegt werden.

Unser langjähriges Vereinsmitglied, Herr Geheimer Bergrat Dr. Heintze, hat bekanntlich durch seine geschirhtlichen Forschungen aus den alten noch vorhandenen Akten Klarbeit darüber geschafft, wie Böttger das „rote Gut", so nannte man diese Art Steinzeug damals, gebrannt hat. Einer der Gebilfen Böttgers, namens David Köhler, hat nämlich liber diese Fabrikation einen Bericht verfaßt, der noch erhalten ist, und dem ich folgendes entnehme:

„Es ist noch mit wenigen des vormahligen gantz Rothen Porcellains

zu gedencken, womit der Anfang gemachet worden ${ }^{\text {... Sie haben aber dem Leim aus dem Blauischen Grunde des }}$ wegen als FluB dem nirrenberger Roth oder der Nirrenberger Erde welche sie auch dem rothen Nürrenberger Stein genennet, zugesetzet, weilen er mit solcher, ein dem Jaspis nicht ungleiches Porcellaine, wenn es poliret gewesen, gegeben. Wie aber derer compositiones gewesen ist dem Gewichte nach nicht bekant, jedoch da sie kleine Öfen gehabt, so haben sie sich jedesmahl mit denen Massen nach dem Grad des Feuers jedes Ofens gerichtet, haben auch unstreitig sehr weiche Masse gehabt. $\mathrm{Zu}$ gedenrken, daB sie nicbt mebr als anderthalb Reihe Geschirre in denen Öfen haben auff ein mahl gutbrennen können, da sie vor denen Mlindungen eine Reihe erst ledig gesetzet, alsdann zwey Reihen mit Geschirre, da aber nicht jedesmabl die andere Reihe gantz gut worden, sie haben drey Virtel von denen Spanbögen probe Lörher in dem Ofen gebabt, da sie spitze Kegel, unten mit drey Füßgen von Leihme aus dem Blauischen Grunde eines Firgers lang unten aber stärcker gemacht in dem Ofen bey dem Probe Loche auff die Capseln gesetzet, wenn diese angefangen, obengläntzend $\mathrm{zu}$ werden und $\mathrm{zu}$ flüßen, darnach haben sie dem Ofen ausgehen laßen ...

. Das Vergltihen geschiehet ordentlich doch kommet es hinten hinaus, zustehen ${ }^{5}$ ). In dem Gutbrennen will eine Capsel zu defendirung vor dem Feuer, daß es nicht zu viel bekomme, dem Geschirre nicht zulänglich sein, sondern es wird ein Schälchen Capsel in ein Spühlnapf Capsel gesetzet, und noch der darzwischen seyende Blatz mit waßer Sande ausgefittert, nachgehends erst das Schälchen auff einem Teckelchen wie sonst gewöhnlich gesetzet in das Schälchen Capsel, und kommen auff denen Seiten dreye hoch ubereinander zu steben, in der Mitten aber viere hoch in jeden Satze der Reihe. Diese Reihe mit rothen Geschirre wird binten an der Stirnmauer unter der Feuer Eße berüber gesetzet, da jedoch sich noch vieles schieff ziehet oder auch wohl gar sich Blatterigt brennet; daß es gut sey, wenn der Ofen ausgegangen ist die Probe, wenn man daran lecket, daß nicht an der Zunge auff einige Maßen anklebe, also noch Feuchtigkeit anziehe, so es nicht mehr an der Zunge anklebet oder anhänget, läßet es sich poliren, welches unumbgänglich seyn muß, weilen es nicht glasuret wird, sondern seinen Glantz und eußerliche Lieblichkeit durch das Schleiffen und Poliren erlanget usw."

Wir benutzen jetzt keinen liegenden Topferofen mehr, wie ihn Böttger zum Brennen seines Steinzeugs und später auch seines Porzellans angewendet bat, aber auch nicht den sonst in der feinkeramischen Industrie viel verbreiteten und zum Brennen weißer Waren vorzüglich bewährten Rundofen, denn auch in einem solchen erbält man beim Brennen von rotem Steinzeug gar leicht ein recht ungleichmäBiges Brenngut, das in den verschiedenen Teilen des Ofens sehr verschieden ausfällt, etwa wie in Fig. 1 schematisch dargestellt.

Es würde also bei Benutzung eines solchen Ofens immer höchstens ein Drittel des eingesetzten Brenngutes ga' gebrannt, dagegen ein Drittel überhitzt und ein Drittel noch matt sein. Diese ungleichmäßige Beschaffenheit der gebrannten Ware in einem Ofen mit direkter Feuerung, d. h. mit durchschlagender Flamme, sei es ein solcher, wie ihn Bobttger hatte, oder ein neuzeitlicber Rundofen, hat ihren Grund vor allem darin, daß die stark eisenhaltige Steinzeugmasse, die hier in Frage kommt, einerseits gegen reduzierende Flammengase sebr empfindlich ist und anderer-eits der GarbrennprozeB, d. h. der eigentliche Erweichungs- und Verglasungsvorgang, ziemlich rasch verläuft, viel rascher z. B. als beim Hartporzellan, das soll Ihnen Fig. 2 schematisch vorführen.

${ }^{5)}$ hinten hinaus, d. h. in den dem Eintritt der Flamme abgekehrten Teil des liegenden halbzylindrischen Brennofens.
Temperalur und Sinterungsgrad sind als Koordinaten eingetragen, und wir erhalten für das Steinzeuy die eine, für das Hartporzellan die andere Brennkurve. Ich betone, daß es sich nur um schematische Darstellungen handelt, denen keine genauen Messungen zugrunde liegen, wenigstens nicht für den hauptsächlichen Verlauf der Kurven. Ich habe sie daher auch punktiert gezeichnet. Die Erfahrung lehrt aber, daß der Verlauf der Kurven in dem Garbrennintervall ungefähr so ist, wie ich dies in dem ausgezogenen Teile der Kurven angedeutet habe, also beim Böttgersteinzeug rasch ansteigend, sich über eine geringe Temperaturspanne erstreckend, beim Porzellan viel allmählicher über einen ausgedehnter en Temperaturbereich verlaufend. Man sagt, die Steinzeugmasse steht infolge ihres Eisen- und Kalkgehaltes im Feuer nicht so gut wie das Porzellan, sowohl hinsichtlich der absoluten Garbrenntemperatur, als auch der Länge ihres Erweichungstemperaturintervalls.

Das Brennen des roten Steinzeugs erfordert also eine besonders erhöhte Aufmerksamkeit und längere Erfahrung, wenn man nicht damit rechnen will, daß ein Teil des Ofeneinsatzes verdirbt, und man brennt es deshalb am besten nur in solchen Ofenkonstruktionen, bei

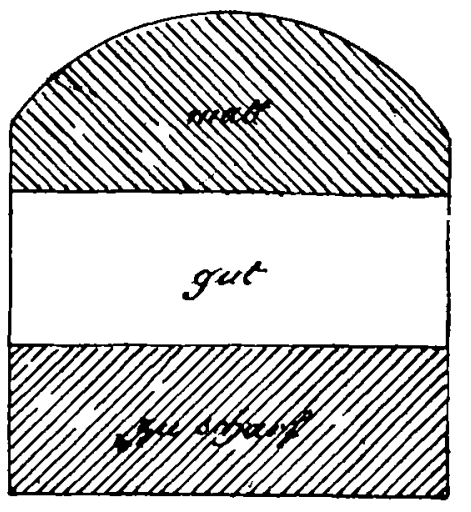

Fig. 1. denen in der Brennkammer jede schädliche Flammenwirkung vermieden und eine gleichmäßige oxydierende Erhitzung des ganzen Ofeninhaltes gewährleistet wird.

Auf diese Weise ist es uns nach Schaffung der erforderlichen Einrichtungen gelungen, seit einem Jahre Geld- und andere Münzen aus solchem rotbraunen Steinzeug flott zu fabrizieren, nicht nur kursfăhiges Geld für Länder und Städte, sondern auch Erinnerungsmedaillen, Wohltätigkeitsmünzen, Reklamemarken u. a. m. Sie haben sich ja

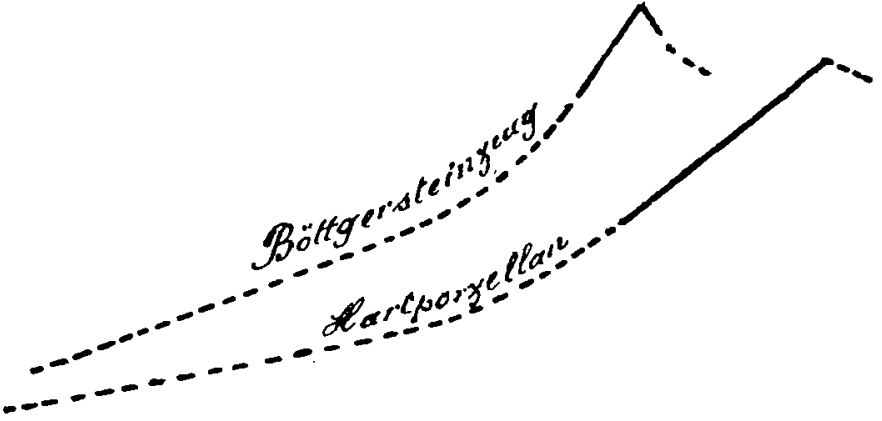

Fig 2.

schon an den in Umlauf gesetzten zahlreichen Sorten von Münzen überzeugen können, für welche verschiedenen Zwecke die neue Münzmasse der Manufaktur Verwendung gefunden hat, und aus welch verschiedenen Kreisen der Bevölkerung sich die Besteller zusammenselzen.

Die sächsischen 20-, 10- und 5-M-Stticke, die nicht kursfähig, sondern nur für Sammelzwecke bestimmt sind, werden mit Goldverzierung versehen; auf Wunsch werden auch andere Münzen vergoldet geliefert. Auch weiße Porzellanmünzen haben wir für verschiedene Besteller auf besonderen Wunsch angefertigt, z. B. Straßenbahnmarken für die Stadt Meißen.

Zum Schlusse will ich noch erwähnen, daß wir diese Bottgersteinzeugmasse in letzter Zeit auch zur Herstellung von Kleinplastiken, Plaketten, Grabmalplatten u. dgl. verwenden. Das Licbtbild eines solchen Grabmales zeige ich herum. Es ist im Auftrage der Schokoladenfabrik Riedel \& Engelmann in Dresden.Plauen angefertigt worden, die es dem Gedächtnis ihrer im Weltkriege gefallenen Arbeiter gewidmet bat. Äbnliche Grabmäler befinden sich unter anderem in einer Meißner Fabrik und an der Kirche in Weißtropp bei Dresden.

Damit habe ich meine Mitteilungen beendet und verweise Sie zum Schlusse noch auf die im Frühjabr 1922 hier in Dresden stattfindende Ausstellung "Deutsche Erden und Steine", wo Sie neben Meißner Porzellan vielleicht auch noch einiges Neue in Böttgersteinzeug ausgestellt sehen werden.

[A. 21.]

\section{Neue Bücher.}

Metallographie. Ausführliches Lehr- und Handhuch der Konstitution und der physikalischen, chemischen und technischen Eigenschaften der Metalle und metallischen Legierungen. Von Prof. Dr. W. Guertler, Dozent an der Techn. Hochschule zu Berlin. 2. Band, 3. Abschnitt: Elektrochemische Metallkunde von Prof. Dr. R. Kremann. XX u. 656 S. Verlag Gebrtider Borntraeger in Berlin W. 1921.

Preis geh. M 210 